\title{
Weighted Multiple Model ADRC for Uncertain Linear Systems
}

\author{
Weicun Zhang ${ }^{*}$, Liuqiao Song ${ }^{2}$, Jing Ge ${ }^{1}$ \\ ${ }^{1}$ School of Automation and Electrical Engineering, University of Science and Technology Beijing, \\ 30 Xueyuan Road Haidian District, Beijing, China \\ E-mail:weicunzhang@ustb.edu.cn \\ www.ustb.edu.cn
}

${ }^{2}$ School of electrical engineering and computer science, Oregon State University,

Corvallis, Oregon OR 97331-5501, USA

\begin{abstract}
For uncertain linear systems such as parameter jumping, this paper presents a weighted multiple model adaptive control frame work, which uses fixed model set to cover the uncertainties of the real plant to be controlled, and for each local model with minor uncertainties, the corresponding local controller is designed based on active disturbance rejection controller (ADRC) approach. Some simulations have been conducted based on MATLAB to verify the effectiveness of the proposed weighted multiple model ADRC adaptive control algorithm.
\end{abstract}

Keywords: Multiple model adaptive control, ADRC, weighting algorithm.

\section{Introduction}

For systems with large uncertainties, such as, model structure change or parameter jumping, conventional adaptive control can no longer meet the performance needs of industrial control systems. Therefore, the control research of uncertain systems has important theoretical significance and practical significance. Weighted multiple model adaptive control (WMMAC) [1-12] can effectively control complex and uncertain systems with prescribed performance indexes. Aiming at the uncertain system, this paper proposes a weighted multiple model adaptive control approach with active disturbance rejection control (ADRC) as local control strategy, which was originally proposed by Professor Jingqing Han [13-20].

Aiming at a class of uncertain linear controlled plants with nonlinear disturbances, this paper combines the active disturbance rejection controller and the weighted multiple model adaptive control system to design a weighted multiple model ADRC control system for the second-order time-delay system and a weighted multiple model ADRC control system for the third-order uncertain system, so that to completed the control of these two uncertain linear systems with nonlinear disturbances. The simulation results show that compared with a single ADRC control system, the weighted multiple model ADRC control system can better meet the control

\footnotetext{
*Corresponding author: W. Zhang, weicunzhang@ustb.edu.cn

(C) The 2021 International Conference on Artificial Life and Robotics (ICAROB2021), January 21 to 24, 2021
} 
performance requirements and has stronger tracking ability and robustness when the parameters are uncertain in a large range or with multiple jumps.

The block diagram of the proposed system is shown in Fig. 1.

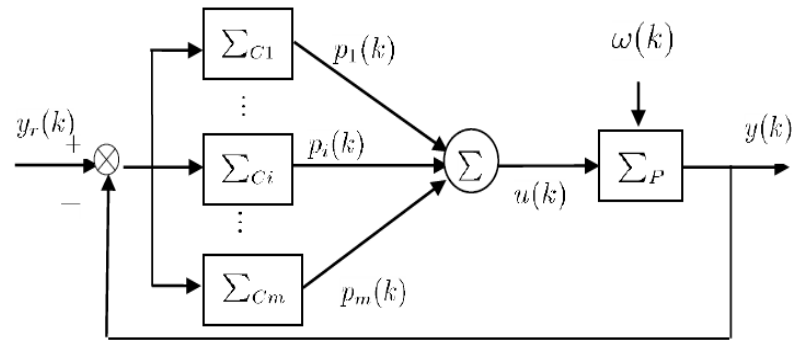

Figure 1 The block diagram of the proposed system

\section{Local controller design based on ADRC}

Consider a plant with small uncertainties

$$
\left\{\begin{array}{c}
\dot{x}_{1}=x_{2} \\
\dot{x}_{2}=x_{3}+b 0 u(t) \\
\dot{x}_{3}=\dot{f}\left(x_{1}, x_{2}, \omega(t), t\right)=h(t) \\
y=x_{1}
\end{array}\right.
$$

where $f($.$) represents unknown unmodeled dynamics or$ 'total disturbance', $u(t)$ control input, $\omega(t)$ external disturbance, $b 0$ control gain, $y(t)$ system output.

First, we need to design the extended state observer (ESO)

$$
\left\{\begin{array}{c}
\varepsilon_{1}=z_{1}-y \\
\dot{z}_{1}=z_{2}-\beta_{01} \varepsilon_{1} \\
\dot{z}_{2}=z_{3}-\beta_{02} \varepsilon_{1}+b_{0} u \\
\dot{z}_{3}=-\beta_{03} \varepsilon_{1}
\end{array}\right.
$$

Its discrete-time version has the following form

$$
\left\{\begin{array}{l}
\varepsilon_{1}=z_{1}(k)-y(k) \\
z_{1}(k+1)=z_{1}(k)+h\left(z_{2}(k)-\beta_{01} \varepsilon_{1}\right) \\
z_{2}(k+1)=z_{2}(k)+h\left(z_{3}(k)-\beta_{02} \varepsilon_{1}+b_{0} u\right) \\
z_{3}(k+1)=z_{3}(k)-h \beta_{03} \varepsilon_{1}
\end{array}\right.
$$

Where $\varepsilon_{1}$ is observation error, $\beta_{01}, \beta_{02}$, and $\beta_{03}$ are the gains of the observer, $z_{1}, z_{2}$, and $z_{3}$ are the estimations of $y, \dot{y}$, and $f($.$) , respectively.$
Second, we need to design the control law based on the ESO

$$
u_{0}=k_{p} e_{1}-k_{d} z_{2}
$$

where $e_{1}=v_{1}-z_{1}, v$ is the reference input of the system, $k_{p}$ and $k_{d}$ are adjustable parameters.

Based on (4), the local control law is given by

$$
u=\frac{u_{0}-z_{3}}{b_{0}}
$$

\section{Multiple model ADRC}

Consider time-varying uncertain plant

$$
\left\{\begin{array}{c}
\dot{x}_{1}=x_{2} \\
\dot{x}_{2}=x_{3}+b(t) u(t) \\
\dot{x}_{3}=\dot{f}\left(x_{1}, x_{2}, d(t), \omega(t), t\right)=h(t) \\
y=x_{1}
\end{array}\right.
$$

Then we need to design multiple model ADRC, which uses multiple models (model set) to cover the uncertainties of $b(t)$, for each (local) model, a local controller (ADRC controller) is designed off-line, and the global control law is weighted sum (on-line) of all the local controllers. That is

$$
u(t)=\sum p_{i}(t) u_{i}(t)
$$

where $u_{i}(\mathrm{t})$ is calculated according to (5).

The weighting algorithm is described as follows (Suppose the number of local controllers is $\mathrm{N}$ )

where $e_{i}$ is the output error of each local model, i.e., $e_{i}(k)=y(k)-y_{i}(k)$. 
The calculation of weights values is carried out at each time instant $k$, and through a ZOH (Zero Order Hold), $p_{i}(t)$ can be obtained from $p_{i}(k)$.

\section{Simulation results}

To verify the effectiveness of the proposed multiple model ADRC framework, many simulations have been conducted with $\omega(t)=0.1 \sin t$ in equation (6), based on MATLAB/ Simulink.

Suppose the plant will change from Model 1 (corresponding to $b(t)=0.6$ in equation (6)) to Model 2 (corresponding to $b(t)=1$ in equation (6)) at time $30 \mathrm{~s}$, and finally change to Model 3 (corresponding to $b(t)=$ 1.5 in equation (6)) at time 60s. The model set include 4 models, i.e., Model1, Model 2, Model 3, and Model 4 (corresponding to $b(t)=2$ in equation (6)). The step responses of multiple model ADRC system and single ADRC system are shown in Fig. 2.

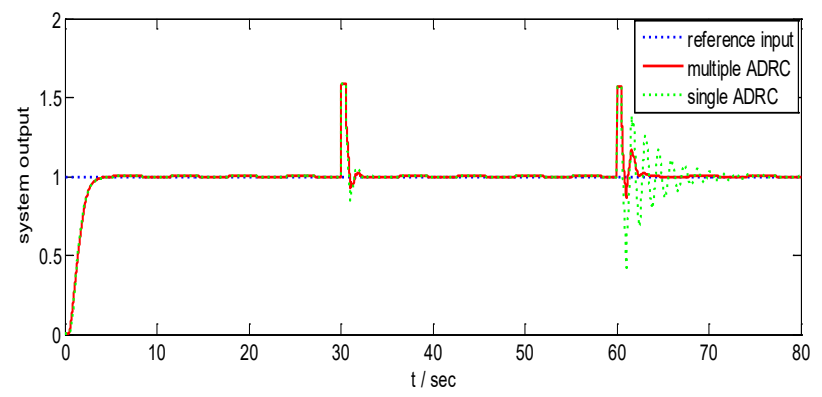

Figure 2. Step response comparison between multiple model ADRC and single ADRC

Weight values of multiple model ADRC are shown in Figure 3.
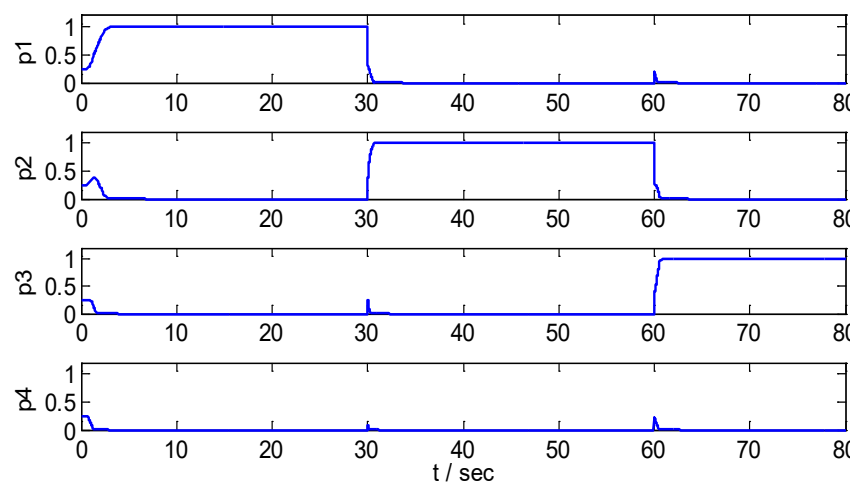

Figure 3. Weight values of multiple model ADRC

\section{Conclusion and future work}

This paper presented a multiple model ADRC framework for uncertain dynamic time-varying system. Simulation results verified the effectiveness of the proposed methods including weighting algorithm and local controller scheme. In the future research work, we will focus on model set selection for time-varying system with more than one variable.

\section{Acknowledgements}

This work was partly supported by National Natural Science Foundation of China (No. 61520106010; 61741302).

\section{References}

1. Fekri, M. Athans and A. Pascoal, Issues, progress and new results in robust adaptive control, International Journal of Adaptive Control and Signal Processing, 2006; 20(10): 519-579

2. D. T. Magill, Optimal adaptive estimation of sampled stochastic processes, IEEE Trans. Automat. Contr., 1965; 10: $434-439$

3. D. G. Lainiotis, Partitioning: A unifying framework for adaptive systems-I: Estimation; II: Control, Proc. IEEE, 1976; 64:1126-1143 and 1182-1197

4. M. Athans et al., The stochastic control of the F-8C aircraft using a multiple model adaptive control (MMAC) method-Part I: Equilibrium flight, IEEE Trans. Automat. Contr., 1977, 22: 768-780

5. A. Badr, Z. Binder, D. Rey, Weighted multi-model control, International journal of systems science, 1992; 23(1): 145149

6. M. Kuipers, P. Ioannou, Practical Robust Adaptive Control: Benchmark Example, Proc. of American Control Conference, Seattle, Washington, USA, 2008:5168-5173

7. Kuipers and P. Ioannou, Multiple model adaptive control with mixing, IEEE Transactions on Automatic Control, 2010; 55(8), 1822-1836

8. S. Baldi, P. A. Ioannou, and E. Mosca, Multiple Model Adaptive Mixing Control: the Discrete-time case, IEEE Trans. on Automatic Control, 2012; 57(4):1040-1045

9. N. Sadati, G. A. Dumont, and H. R. Feyz Mahdavian, Robust Multiple Model Adaptive Control Using Fuzzy Fusion, 42nd South Eastern Symposium on System Theory, Tyler, TX, USA, March 7-9, 2010

10. Z. Han, and K. S. Narendra, New Concepts in Adaptive Control Using Multiple Models, IEEE Transactions on Automatic Control, 2012; 57(1): 78-89

11. W. Zhang, Stable Weighted Multiple Model Adaptive Control: Discrete-Time Stochastic Plant, International Journal of Adaptive Control and Signal Processing, 2013; 27(7): 562-581

(C) The 2021 International Conference on Artificial Life and Robotics (ICAROB2021), January 21 to 24, 2021 
12. W. Zhang, Further Results on Stable Weighted Multiple Model Adaptive Control: Discrete-Time Stochastic Plant, International Journal of Adaptive Control and Signal Processing, 2015; 29(12): 1497-1514

13. J. Han. Active disturbance rejection control technique-the technique for estimating and compensating the uncertainties. Beijing: National Defense Industry Press; 2008.

14. J. Han. From PID to active disturbance rejection control. IEEE Transactions on Industrial Electronics 2009; 56(3): 900-906.

15. Aaron Radke, and Z. Gao. A survey of state and disturbance observers for practitioners, Porc. American Control Conference. 2006 5183-5188.

16. Y. Huang, W.C. Xue. Active disturbance rejection control: Methodology and theoretical analysis. ISA Transactions 2014; 53: 963-976.
17. Wenchao Xue, Wenyan Bai, Shen Yang, Kang Song, Yi Huang, and Hui Xie. ADRC with adaptive extended state observer and its application to air-fuel ratio control in gasoline engines. IEEE Transactions on Industrial Electronics 2015; 62: 5847-5857.

18. Z. Gao. Active disturbance rejection control: a paradigm shift in feedback control system design. Proc. American Control Conference. 2006 2399-2405.

19. Qing Zheng, Linda Q. Gao, \& Zhiqiang Gao. On stability analysis of active disturbance rejection control for nonlinear time-varying plants with unknown dynamics. Proc. IEEE Conference on Decision and Control. 2007 3501-3506.

20. Qing Zheng, Zhongzhou Chen, \& Zhiqiang Gao. A practical approach to disturbance decoupling control. Control Engineering Practice 2009; 17: 1016-1025. 